\title{
Laboratory Astrochemistry: A Powerful Tool to Understand the Origin of Organic Molecules in the Interstellar Medium, Comets, and Meteorites
}

\author{
Michel Nuevo*t \\ NASA Ames Research Center, Moffett Field, CA, USA \& SETI Institute, Mountain View, CA, USA \\ E-mail: michel.nuevo-1@nasa.gov
}

During the past two decades, astrochemistry laboratory simulations have shown that complex organic molecules can be formed under simulated astrophysical conditions from the vacuum ultraviolet (UV) irradiation of ice mixtures containing simple species such as $\mathrm{H}_{2} \mathrm{O}, \mathrm{CO}, \mathrm{CO}_{2}$, $\mathrm{CH}_{3} \mathrm{OH}$, and $\mathrm{NH}_{3}$. These organics include compounds of biological and prebiotic interests such as amino acids - the building blocks of proteins, and nucleobases - the informational subunits of DNA and RNA. Although the presence of amino acids in the interstellar medium (ISM) has not been confirmed by observations to date, they have been detected in meteorites, indicating that biomolecules and/or their precursors can be formed under extraterrestrial, non-biological conditions. Nucleobases have also been detected in meteorites, broadening the variety of complex organic molecules that can be formed in astrophysical environments. Like amino acids, nucleobases and other $N$-heterocycles have not been observed in the ISM. In the following, I will review some of the progress made by laboratory astrochemistry towards understanding the formation of organic species from the UV irradiation of ices at low temperature under astrophysically relevant conditions. This discussion will be focused on the formation of amino acids and other molecules of prebiotic interest such as urea and glycerol. Then, I will present recent studies on the formation of nucleobases and related compounds from the UV irradiation of pyrimidine in $\mathrm{H}_{2} \mathrm{O}$, $\mathrm{NH}_{3}$, and $\mathrm{CH}_{3} \mathrm{OH}$ ices, which show the formation of a large suite of photo-products including the nucleobases uracil and cytosine.

Frank N. Bash Symposium New Horizons In Astronomy,

October 9-11, 2011

Austin Texas

* Speaker.

${ }^{\dagger}$ With the collaboration of, in an alphabetical order: G. Auger, D. Blanot, J.H. Bredehöft, Y.-J. Chen, G.D. Cody, E. Dartois, B.T. De Gregorio, D. Deboffle, J.P. Dworkin, J.E. Elsila, W.-H. Ip, A.L.D. Kilcoyne, L. Le Sergeant d'Hendecourt, P. de Marcellus, C.K. Materese, U.J. Meierhenrich, S.N. Milam, G.M. Muñoz Caro, L. Nahon, S.A. Sandford, W.H.-P. Thiemann, C.-Y.R. Wu, and T.-S. Yih. 


\section{Ices and organics in the Solar System and the interstellar medium}

Gas (atoms, molecules, ions) and dust grains (solid particles) in the interstellar medium (ISM) are clumped into clouds and processed by energetic particles, mainly photons (ultraviolet, X-rays, $\gamma$-rays) and cosmic rays (high energy protons and other heavier nuclei). Given sufficient mass, the gravitational collapse of these clouds will eventually lead to the formation of stars via several stages of evolution, from diffuse clouds to denser molecular clouds, and finally to protostellar objects surrounded by debris disks.

Dust grains have a typical size of $\sim 0.1 \mu \mathrm{m}$ and are usually made of carbonaceous material $[1,2]$ or minerals (amorphous or crystalline) [3, 4]. In cold environments such as interstellar dense clouds and inside disks around protostars, grains are coated with a volatile mantle consisting of ices. Here the term "ice" refers to any volatile species that can condense on cold grains, mainly $\mathrm{H}_{2} \mathrm{O}, \mathrm{CO}, \mathrm{CO}_{2}, \mathrm{CH}_{3} \mathrm{OH}, \mathrm{NH}_{3}$, and $\mathrm{CH}_{4}$ [5-10], but they can also be other more complex organic molecules, including polycyclic aromatic hydrocarbons (PAHs) [11 - 13]. Icy compounds are usually bound together by Van der Waals interaction and/or hydrogen bonds, and participate actively to the chemical and thermal coupling between the grains and the surrounding gas.

Ices are ubiquitous in the ISM, and present in the (outer) Solar System (Table 1). It first appears that the composition of astrophysical ices is comparable in astrophysical objects and in comets. The most abundant component of astrophysical ices is $\mathrm{H}_{2} \mathrm{O}$. Small carbon- and oxygenbearing molecules such as $\mathrm{CO}, \mathrm{CO}_{2}$, and $\mathrm{CH}_{3} \mathrm{OH}$ have abundances ranging from a few percent to $30 \%$ compared with $\mathrm{H}_{2} \mathrm{O} . \mathrm{NH}_{3}$ is not very abundant, but its photochemistry in the presence of the other ices leads to the formation of new species including $\mathrm{OCN}^{-}$, observed in many astrophysical ices with detectable abundances [14].

The interaction of the ices with ultraviolet (UV) photons and cosmic rays modifies the chemical structure of the mantle. As more and more complex molecules are formed, an organic refractory layer starts to form on the grains. Experimental simulations have shown that such a refractory material, often called organic residue, can be formed from the irradiation of astrophysically relevant ice mixtures with UV photons or high-energy protons [15-17]. Stable at room temperature, residues can then be analyzed with several techniques.

These complex organic molecules are then probably incorporated into small objects during the formation of the protoplanetary disk and the stellar system, such as comets, asteroids, and interplanetary dust particles (IDPs). The bombardment of planets from comets and asteroids during the heavy bombardment period in the early stages of the formation of our Solar System may have seeded the primitive Earth, as well as other planets, with a broad range of organic molecules, including the ingredients necessary for the emergence of life as we know it $[18,19]$. These ingredients are amino acids (the building blocks of proteins), nucleobases (the informational subunits of DNA and RNA), ribose (the carbonaceous backbone of DNA and RNA), as well as other sugars, and lipids (constituents of cell walls). The detection of these compounds in meteorites [20-22] strongly supports an extraterrestrial origin of the building blocks of life.

In the following, I will review what is known about the composition of organic residues formed from the UV irradiation of astrophysically relevant ices. I will discuss the detection and formation of amino acids and nucleobases in these residues, and compare those results with what has been observed in meteorites and collected extraterrestrial materials. 
Table 1: Composition and relative abundances of ices in the interstellar medium and comparison with comets. The abundance of $\mathrm{H}_{2} \mathrm{O}$, most abundant ice component, has been set to 100 for all these objects.

\begin{tabular}{cccccc}
\hline & RAFGL 7009S & NGC 7538 IRS9 & W33A & Elias 16 & Comets \\
Species & {$[23]$} & {$[24,25]$} & {$[5]$} & {$[5]$} & {$[5]$} \\
\hline \hline $\mathrm{H}_{2} \mathrm{O}$ & 100 & 100 & 100 & 100 & 100 \\
$\mathrm{CO}$ & 15 & 12 & 8 & 25 & $5-3$ \\
$\mathrm{CO}_{2}$ & 21 & 15 & 13 & 18 & $3-20$ \\
$\mathrm{CH}_{3} \mathrm{OH}$ & 30 & $4-12$ & 18 & $<3$ & $0.3-5$ \\
$\mathrm{CH}_{4}$ & 3.6 & 2 & 0.4 & - & 1 \\
$\mathrm{NH}_{3}$ & - & 13 & 15 & $\leq 9$ & $0.1-1.8$ \\
$\mathrm{OCN}^{-}$ & 3.7 & 2 & 3.5 & $<0.5$ & - \\
\hline
\end{tabular}

\section{Laboratory simulations}

Mixtures of the compounds to be studied are prepared in the gas phase in a stainless steel or glass mixing line (background pressure: $\leq 10^{-5}$ mbar). Relative abundances between components are determined by their partial pressures. Gas mixtures are transferred into bulbs, which are then connected to the deposition tube of a vacuum chamber. Vacuum chambers consist of a stainless steel cage pumped to a pressure of a few $10^{-8}$ mbar and cooled to temperatures as low as $10 \mathrm{~K}$ with a closed-cycle He cryocooler. A detailed description of typical vacuum system set-ups can be found in refs. [26-28].

Mixtures are then simultaneously deposited on a cold substrate (infrared-transparent $\mathrm{KBr}$ or $\mathrm{MgF}_{2}$ windows, aluminum, etc.) and irradiated with UV photons or energetic protons. UV photons can be provided either by a microwave-powered $\mathrm{H}_{2}$-discharge lamp, emitting mainly Lyman- $\alpha$ and a continuum centered around $160 \mathrm{~nm}$ [27-30], or by a synchrotron radiation beam (tunable light source), provided by facilities such as former LURE (Orsay, France) [31], recently open SOLEIL (Gif-sur-Yvette, France) [32], or NSRRC (Hsinchu, Taiwan) [26, 33]. UV lamp radiation is a good analog to the light emitted by young stars, whereas UV light from synchrotron can be used to study a given photochemical reaction or pathway at a specific wavelength. Ices can also be bombarded with energetic protons produced by a Van de Graaff accelerator [34]. The deposition/irradiation duration can vary from a few minutes to a few days, with typical experiments lasting 1 to 2 days. After irradiation, samples are usually warmed to room temperature, at which time they are recovered from their substrate and conserved before they are analyzed with different techniques. Some vacuum systems are also equipped with a Fourier-transform infrared (FTIR) spectrometer in order to monitor the composition of the ices during irradiation and warm-up. The infrared (IR) spectra obtained from such experiments can in some cases be directly compared with astronomical data in order to search for the presence of ice components (see, e.g., refs. [35, 36]) and/or the physical conditions in the media where those species are observed.

\section{Composition of organic residues}

Infrared spectroscopy is a powerful tool to monitor the evolution of the ices during irradiation at low temperature and warm-up to room temperature. UV irradiation of ices induces the breaking 

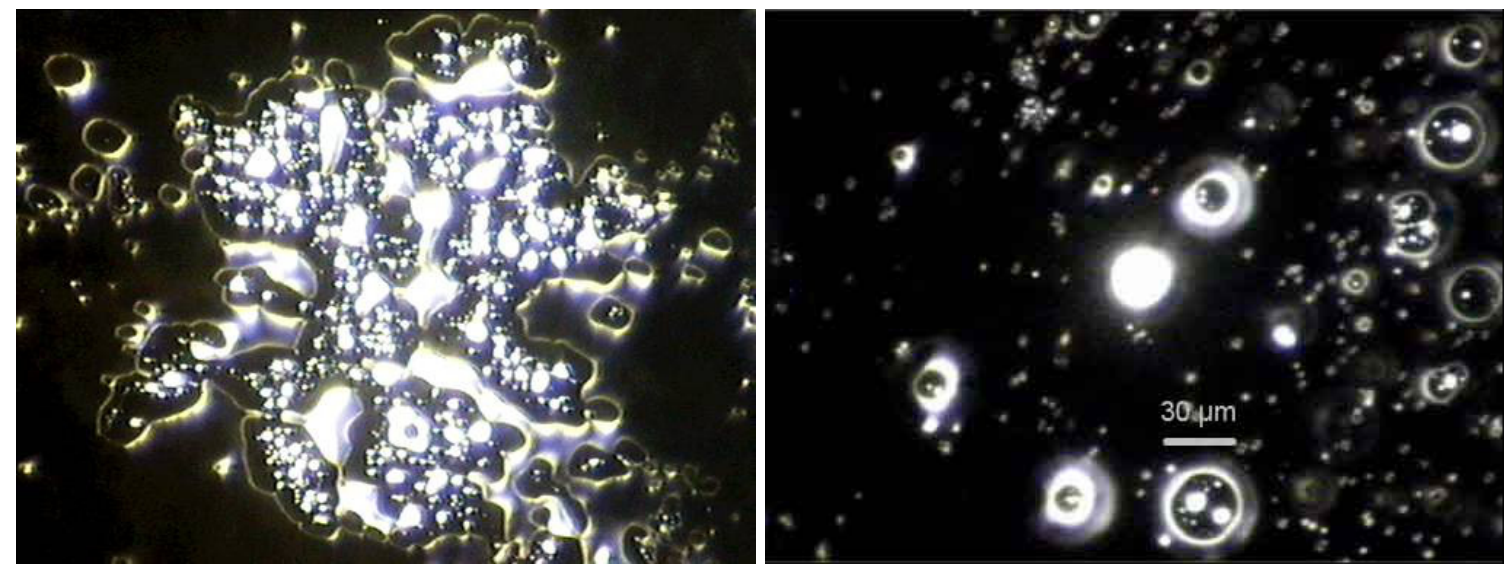

Figure 1: Infrared microscopy images of an organic residue produced from the UV irradiation of an $\mathrm{H}_{2} \mathrm{O}: \mathrm{CH}_{3} \mathrm{OH}: \mathrm{NH}_{3}$ ice mixture. Organic molecules form vesicle-like structures that are soluble in water.

of chemical bonds and/or the ionization of some compounds, leading to the formation of reactive species such as ions and radicals. The mobility of radicals in an ice matrix is usually very limited at low temperature, and only first-opportunity reactions between nearby species can take place at this stage. After irradiation and during warm-up, photo-produced species become more mobile and react with each other to form more complex molecules. IR spectra at low temperature of photolyzed ice mixtures containing $\mathrm{H}_{2} \mathrm{O}$ and combinations of $\mathrm{CO}, \mathrm{CO}_{2}, \mathrm{CH}_{3} \mathrm{OH}, \mathrm{CH}_{4}$, and $\mathrm{NH}_{3}$ indicate the presence of several families of molecules including alcohols, amines, carboxylic acids, alkanes, and alkenes, as well as species such as $\mathrm{H}_{2} \mathrm{CO}$ (formaldehyde), $\mathrm{NH}_{4}^{+}$(ammonium ion), $\mathrm{OCN}^{-}$(cyanate ion, observed in astrophysical objects, see Table 1), the $\mathrm{HCO}^{\bullet}$ radical, $\mathrm{HNCO}$ (isocyanic acid), and organic molecules as complex as $\mathrm{NH}_{2} \mathrm{CHO}$ (formamide) [26, 31, 37].

During the warm-up phase, all volatile species (both starting ices and new photo-products), sublime away from the substrate. Some of those volatile compounds can be identified by mass spectrometry, such as carbamic acid, produced from the irradiation of an $\mathrm{H}_{2} \mathrm{O}: \mathrm{CO}_{2}: \mathrm{NH}_{3}$ mixture, and observed to sublime around $250 \mathrm{~K}$ [26].

At room temperature, organic residues usually appear oil-like with a flavescent tinge. They are sometimes referred to as "yellow stuff", and are soluble in water $[16,38]$. They usually consist of $10-50 \mu \mathrm{m}$ droplets (Fig. 1), suggesting that a decent fraction of the formed photo-products can selfassemble into vesicle structures [39]. Laser-desorption mass spectrometry of one residue showed a very complex mass spectrum displaying a peak for almost every single mass from 40 to 400 atomic mass units (amu), indicating that this material is very complex and probably macromolecular [40].

IR spectra of organic residues show a number of characteristic bands assigned to molecular families including alcohols, amines, carboxylic acids, alkanes, alkenes, nitriles/isonitriles, ketones, esters, and amides, as well as carboxylate salts [31,37]. Specific compounds could not be identified in residues, with the exception of hexamethylenetetramine (HMT) [17] and $\mathrm{NH}_{4}^{+}$[41].

More recently, X-ray absorption near-edge structure (XANES) spectra of similar residues have been measured in the carbon, oxygen, and nitrogen edges at the Advanced Light Source (Berkeley, USA) [42]. These measurements confirmed the presence of several families of chemical groups (aromatic and aliphatic $\mathrm{CH}_{\mathrm{x}}$ bonds, carboxyls, amides, ketones, nitriles, etc.). This study 
also indicated that oxygen and nitrogen atoms are very efficiently incorporated into carbonaceous chains, resulting in a very nitrogen- and oxygen-rich material, with elemental N/C and O/C ratios of 0.1-0.3 and 0.4-0.6, respectively [42]. Compared with XANES measurements of extraterrestrial materials, the N/C and $\mathrm{O} / \mathrm{C}$ ratios found in organic residues are up to 2 times higher than what was measured in cometary particles returned by Stardust [43, 44], and 2-10 times higher than for organic matter extracted from the Murchison meteorite [45, 46].

However, mass spectrometry as well as IR and X-ray absorption spectroscopies techniques are usually not sufficient to identify specific organic molecules. For this, residues need to be analyzed with chemical techniques such as liquid and gas chromatographies.

\section{Amino acids in organic residues}

The first organic molecules that were searched for in residues as well as in carbonaceous chondrites were amino acids. Amino acids are the building blocks of proteins for all life on Earth. There are 20 proteinic amino acids, plus 2 additional rare ones - selenocysteine and pyrrolysine - that were found in some proteins. Other non-proteinic amino acids can also be found in biological systems, such as sarcosine or $\beta$-alanine [47]. In proteins, amino acids are only present in their enantiomeric form $\mathrm{L}$. This property of homochirality is chemically favored for amino acid polymerization, and gives a particular chiral 3-dimensional structure. D-Amino acids, such as D-alanine and D-aspartic acid, are also found in some biological systems such as bacteria cell membranes [47].

Organic residues produced from the UV irradiation of ice mixtures containing $\mathrm{H}_{2} \mathrm{O}, \mathrm{CO}, \mathrm{CO}_{2}$, $\mathrm{CH}_{3} \mathrm{OH}, \mathrm{CH}_{4}$, and $\mathrm{NH}_{3}$, and analyzed with high-performance liquid chromatography (HPLC) and/or gas chromatography coupled with mass spectrometry (GC-MS) showed the presence of a broad variety of amino acids [27, 29], up to 16 in one sample [30]. These amino acids are mostly detected after acid hydrolysis of the residues, as only very small quantities of free amino acids are present in non-hydrolyzed residues [27], indicating that organic residues consist of either a macromolecular material that can be easily hydrolyzed, or precursors which are more photo-stable to UV photons than the compounds detected after hydrolysis, or both.

Because they are formed from achiral starting compounds under achiral experimental conditions, amino acids detected in residues are racemic, i.e., they have equal amounts of D- and L-enantiomers [29, 30, 48]. In addition, the distribution of amino acids is different from what is observed in biological proteins, as the most abundant amino acid—glycine—is also the simplest, and the abundance of larger amino acids decreases with their molecular weight [27, 48]. Finally, the formation of amino acids from the UV irradiation of astrophysical ice analogs has proven efficient as long as the starting ices contain $\mathrm{C}, \mathrm{H}, \mathrm{N}$, and $\mathrm{O}$ atoms, regardless of the starting carbon source [27]. For example, amino acids have been detected in residues formed from the UV irradiation of a fully inorganic starting mixture $\mathrm{H}_{2} \mathrm{O}: \mathrm{CO}_{2}: \mathrm{NH}_{3}$ [33], as well as from the UV/EUV irradiation of naphthalene $\left(\mathrm{C}_{10} \mathrm{H}_{8}\right)$, the smallest $\mathrm{PAH}$, in an $\mathrm{H}_{2} \mathrm{O}: \mathrm{NH}_{3}$ ice mixture [49]. These characteristics are consistent with non-biological formation pathways, and indicate that the amino acids detected in residues are not due to biological contamination.

Amino acids have been extensively searched for in the ISM in the gas phase (radio astronomy) [50], but none of them have been detected, though the possible detection of glycine is still under debate [51, 52]. In contrast, up to 70 amino acids have been found in carbonaceous 
chondrites such as the Murchison and Murray meteorites [20, 53, 54]. These meteoritic amino acids, of which only a small fraction are found in proteins, have isotopic ratios consistent with an extraterrestrial origin [55] and a non-racemic distribution, with enantiomeric excesses of a few percent for proteinic-like $\alpha$-hydrogenated amino acids [21, 56, 57], and up to $18 \%$ for isovaline, a non-proteinic $\alpha$-methylated amino acid [58, 59].

The origin of this asymmetry in the chiral distribution of amino acids is still a mystery, and a number of theoretical and experimental studies have attempted to reproduce such a result by adding asymmetry to the formation of organic residues from the UV irradiation of ices [31]. Recently, the irradiation of $\mathrm{H}_{2} \mathrm{O}: \mathrm{CH}_{3} \mathrm{OH}: \mathrm{NH}_{3}$ (achiral) ice mixtures with circularly polarized light (CPL) in the UV range has shown that alanine, the smallest chiral proteinic amino acid, was formed with enantiomeric excesses of opposite signs when left or right polarizations of the light were used [32]. Moreover, enantiomeric excesses seem to be directly proportional to the number of polarized photons irradiating the ices. Astronomical observations showed that CPL could be present in the ISM and affect the stereochemistry of carbonaceous molecules formed in astrophysical environments $[60,61]$.

\section{Other molecules of prebiotic interest in organic residues}

Although present in all organic residues formed from the UV irradiation of simple astrophysical ice analogs $\left(\mathrm{H}_{2} \mathrm{O}, \mathrm{CO}, \mathrm{CO}_{2}, \mathrm{CH}_{3} \mathrm{OH}, \mathrm{CH}_{4}, \mathrm{NH}_{3}\right.$, etc. $)$, amino acids only represent a small fraction of the residue material, with a quantum yield estimated to be of the order of $10^{-4}$ for the total amount of amino acids [30]. Other molecules of prebiotic interest, in particular DNA- and RNAlike nucleobases, have been searched for in organic residues, with no success.

Nonetheless, molecules such as urea, glycerol, and a number of its derivatives including glyceric amide, glycolic acid, and glycerolic acid [62] have been detected in residues. These compounds are known to perform different functions in biological systems, and some of them have been detected in meteorites [22, 63, 64]. The presence of these compounds in residues has been confirmed in other experiments, which also showed the presence of hydantoin [65]. This compound, also detected in meteorites [64, 66], is particularly interesting because its hydrolysis leads to the formation of carbamoyl amino acids (CAAs), which can also be formed from the addition of isocyanic acid (HNCO) to $\alpha$-amino acids. CAAs are the precursors of $N$-carboxyanhydride amino acids, which can polymerize into poly- and oligopeptides, that is, primitive proteins [67, 68].

Finally, amphiphilic molecules, consisting of a hydrophilic head and a hydrophobic tail, are also present in organic residues (see Fig. 1 and ref. [39]). These compounds have the ability of selfassembling into vesicles. The delivery of amphiphiles via meteorites and comets to the primitive Earth may have led to the formation of the first cell membranes, within which chemical reactions are protected from the outside environment [69].

Future analysis of organic residues will focus on the search for sugar compounds, which are assumed to be present, but whose presence in residues has never been demonstrated, though they have been detected in the Murchison meteorite [22]. 


\section{Formation of nucleobases from pyrimidine in astrophysical ices}

Nucleobases, the informational subunits of DNA and RNA, are compounds based on the backbones of two $N$-heterocyclic compounds, namely, pyrimidine and purine. Biological pyrimidinebased nucleobases are uracil (RNA), thymine (DNA), and cytosine (DNA/RNA), whereas biological purine-based nucleobases are adenine and guanine, both found in DNA and RNA. There are also other pyrimidine- and purine-based compounds in the nature including barbituric acid, its derivatives, and caffein.

Nucleobases have been extensively searched for in the ISM in the gas phase [70 - 72], but never been detected. Only an upper limit for the column density of pyrimidine of $1.7-3.4 \times 10^{14} \mathrm{~cm}^{-2}$ could be derived from these observations [71]. More generally, no $N$-heterocyclic molecules have been unequivocally detected so far in the ISM [72], although there are observational indications that they may be present [73]. However, purines have been found in a large number of carbonaceous chondrites including Murchison, Murray, and Orgueil [74-77]. Meteoritic pyrimidines are usually detected with smaller abundances than purines because they are more subject to chemical and photochemical processes. Nonetheless, uracil was found in water extracts of Murchison, Murray, and Orgueil [78]. Finally, the extraterrestrial origin of nucleobases in Murchison has been confirmed by isotopic measurements [79].

Therefore, pyrimidines and purines must be present in astrophysical environments. Since they are not observed in the gas phase, they may be condensed on the surface of cold grains, mixed with ices, together with other aromatic molecules [11, 12]. Moreover, some theoretical studies suggest that pyrimidine and other aromatic compounds could be formed via polymerization of smaller molecules [80]. For this reason, experiments in which pyrimidine is mixed with ices of astrophysical interest and irradiated with UV photons has been and are currently being performed [28, 81].

The UV irradiation of pyrimidine mixed with pure $\mathrm{H}_{2} \mathrm{O}$ ice leads to the formation of an organic residue in which several pyrimidine derivatives have been detected, including the nucleobase uracil and its precursor 4(3H)-pyrimidone [28]. Theoretical calculations simulating such reactions are in agreement with experimental results, and showed that $4(3 H)$-pyrimidone and uracil are the most stable singly and doubly oxidized derivatives formed, respectively [82]. These calculations also showed that $\mathrm{H}_{2} \mathrm{O}$ plays an essential as an ice matrix to stabilize the formation of these photoproducts.

The photochemistry of pyrimidine mixed other pure ices such as $\mathrm{NH}_{3}$ (ammonia), $\mathrm{CH}_{3} \mathrm{OH}$ (methanol), or $\mathrm{CH}_{4}$ (methane) is not as efficient, although a few singly substituted pyrimidine derivatives do form, such as 4-aminopyrimidine in $\mathrm{NH}_{3}+$ pyrimidine mixtures [81], or 4-pyrimidinemethanol in $\mathrm{CH}_{3} \mathrm{OH}+$ pyrimidine mixtures (unpublished results). The addition of $\mathrm{H}_{2} \mathrm{O}$ in those mixtures not only diversifies the chemistry by oxidizing pyrimidine and its amino derivatives, but it also increases the efficiency of addition of $\mathrm{NH}_{2}, \mathrm{CH}_{2} \mathrm{OH}$, and $\mathrm{CH}_{3}$ groups to pyrimidine, probably via the same matrix effect as observed for $\mathrm{H}_{2} \mathrm{O}+$ pyrimidine mixtures [82]. In $\mathrm{H}_{2} \mathrm{O}$-rich mixtures, identified photo-products include the nucleobases uracil and cytosine, as well as small quantities of non-aromatic species such as glycine, urea, and small amino acids [81]. However, the addition of $\mathrm{CH}_{3}$ groups to pyrimidine appears to be an inefficient process. Consequently, thymine, the third pyrimidine-based nucleobase, can only form when the number of UV photons per deposited molecule is significantly higher (unpublished results). 
Future experiments will focus on the study of the irradiation of pyrimidine in a realistic mixture of astrophysical ice analogs, in order to assess the role of each ice component in the formation of pyrimidine derivatives, including nucleobases and their isomers.

\section{Laboratory astrochemistry, extraterrestrial materials, and space missions}

IR studies of ice mixture irradiations can be directly compared with IR astronomical observations of a broad range of astrophysical objects from ISO (European Space Agency, ESA) and Spitzer (NASA). For instance, laboratory experiments helped identifying the interstellar bands of $\mathrm{OCN}^{-}[83,84]$ and $\mathrm{NH}_{3}[35,36]$. On the other hand, techniques used to analyzed organic residues produced in the laboratory are usually applicable to the analysis of extraterrestrial materials such as meteorites, IDPs, and samples returned by space missions, for a direct comparison.

For example, cometary grains collected by Stardust from comet Wild 2 and returned to Earth have been analyzed with IR and XANES spectroscopies, as well as liquid and gas chromatographies. Results show that the composition of these grains varies significantly from one to another, displaying a broad range of organic compounds [43, 44, 85]. These measurements can be directly compared with what is observed for IDPs [86] and laboratory organic residues [42], and constrain the conditions in which those materials form in astrophysical environments [87]. NASA is already planning a future sample-return mission (OSIRIS-REx) currently scheduled for launch in 2016 for an encounter with asteroid 1999 RQ36 in 2023.

Similar analyses for the search of organics will probably be performed on the grains returned by Hayabusa from asteroid Itokawa, although mineralogical analysis showed that this S-type asteroid has the same IR characteristic features as LL ordinary chondrites [88], known to be poor in carbonaceous matter. The Japanese space agency (JAXA) is currently working on Hayabusa 2.

Analysis of the fragments of the Almahata Sitta meteorite, collected in Sudan after asteroid $2008 \mathrm{TC}_{3}$ entered the Earth's atmosphere [89], showed that this unique ureilite contains organic compounds including amino acids $[90,91]$. Almahata Sitta was also found to contain adenine, one of the purine nucleobases [77]. Further analysis of these meteoritic fragments may be conducted in order to identify other organic compounds of prebiotic interest.

Finally, the Rosetta mission (ESA), launched in 2004 [92], will encounter comet ChuryumovGerasimenko and land the Philae probe on its nucleus in 2014, where several in-situ analytical techniques will be performed. Instruments onboard Philae include a GC-MS instrument which can automatically prepare and run samples [93]. These data will be directly comparable with laboratory measurements of organic residues, meteoritic samples, IDPs, and Stardust grains.

\section{Conclusion}

Laboratory astrochemistry is a rich, powerful research field in constant evolution. Laboratory experiments can constrain physical and chemical parameters in observed astrophysical environments, while astronomical observations can test laboratory predictions. In addition, analytical techniques used to analyze samples produced in the laboratory are in most cases applicable to the study of extraterrestrial materials such as meteorites, IDPs, and samples returned from Solar 
System objects such as comets and asteroids. Direct comparison between these data is a powerful tool to assess the composition, the origin, and the evolution of extraterrestrial matter, as well as the link between astronomical observations and extraterrestrial material collected on Earth. In particular, one of the goals of astrochemistry is to study the evolution and origin of molecules of biological interest, from the astrophysical environments where they form, to their incorporation into Solar System bodies and their delivery to telluric planets such as the primitive Earth.

\section{References}

[1] A. Blanco, S. Fonti, \& V. Orofino, On the interstellar extinction hump and laboratory carbonaceous grains, Astrophys. J. 462 (1996) 1020.

[2] E. Dartois, Interstellar dust grains: the hydrogenated amorphous carbon contribution, proceedings of Molecules in Space and Laboratory, Paris, France, 14-18 May 2007, J.L. Lemaire \& F. Combes, Eds. (2007) p. 54.

[3] D.M. Watson, J.M. Leisenring, E. Furlan, et al., Crystalline silicates and dust processing in the protoplanetary disks of the Taurus young cluster, Astrophys. J. Supp. 180 (2009) 84.

[4] I. Oliveira, J. Olofsson, K.M. Pontoppidan, et al., On the evolution of dust mineralogy, from protoplanetary disks to planetary systems, Astrophys. J. 734 (2011) 51.

[5] E.L. Gibb, D.C.B. Whittet, W.A. Schutte, et al., An inventory of interstellar ices toward the embedded protostar W33A, Astrophys. J. 536 (2000) 347.

[6] E. Dartois, The ice survey opportunity of ISO, Space Sci. Rev. 119 (2005) 293.

[7] A.C.A. Boogert, K.M. Pontoppidan, C. Knez, C., et al., The c2d Spitzer spectroscopic survey of ices around low-mass young stellar objects. I. $\mathrm{H}_{2} \mathrm{O}$ and the 5-8 $\mu \mathrm{m}$ bands, Astrophys. J. 678 (2008) 985.

[8] K.M. Pontoppidan, A.C.A. Boogert, H.J. Fraser, et al., The c2d Spitzer spectroscopic survey of ices around low-mass young stellar objects. II. CO 2 , Astrophys. J. 678 (2008) 1005.

[9] K.I. Öberg, A.C.A. Boogert, K.M. Pontoppidan, et al., The c2d Spitzer spectroscopic survey of ices around low-mass young stellar objects. III. CH, Astrophys. J. 678 (2008) 1032.

[10] S. Bottinelli, A.C.A. Boogert, J. Bouwman, et al., The c2d Spitzer spectroscopic survey of ices around low-mass young stellar objects. IV. $\mathrm{NH}_{3}$ and $\mathrm{CH}_{3} \mathrm{OH}$, Astrophys. J. 718 (2010) 1100.

[11] S.A. Sandford, M.P. Bernstein, \& L.J. Allamandola, The mid-infrared laboratory spectra of naphthalene $\left(\mathrm{C}_{10} \mathrm{H}_{8}\right)$ in solid $\mathrm{H}_{2} \mathrm{O}$, Astrophys. J. 607 (2004) 346.

[12] M.P. Bernstein, S.A. Sandford, \& L.J. Allamandola, The mid-infrared absorption spectra of neutral polycyclic aromatic hydrocarbons in conditions relevant to dense interstellar clouds, Astrophys. J. Suppl. Ser. 161 (2005) 53.

[13] V.C. Geers, E.F. van Dishoeck, R. Visser, R., et al., Spatially extended polycyclic aromatic hydrocarbons in circumstellar disks around T Tauri and Herbig Ae stars, Astron. Astrophys. 476 (2007) 279.

[14] R.J.A. Grim \& J.M. Greenberg, Ions in grain mantles - The 4.62 micron absorption by $\mathrm{OCN}^{-}$in W33A, Astrophys. J. 321 (1987) L91.

[15] W. Hagen, L.J. Allamandola, \& J.M. Greenberg, Interstellar molecule formation in grain mantles The laboratory analog experiments, results and implications, Astrophys. Space Sci. 65 (1979) 215. 
[16] V.K. Agarwal, W. Schutte, W., J.M. Greenberg, et al., Photochemical reactions in interstellar grains Photolysis of $\mathrm{CO} / \mathrm{NH}_{3}$ and $\mathrm{H}_{2} \mathrm{O}$, Orig. Life 16 (1985) 21.

[17] M.P. Bernstein, S.A. Sandford, L.J. Allamandola, \& S. Chang, Organic compounds produced by photolysis of realistic interstellar and cometary ice analogs containing methanol, Astrophys. J. $\mathbf{4 5 4}$ (1995) 327.

[18] J. Oró, Comets and the formation of biochemical compounds on the primitive Earth, Nature 190 (1961) 389.

[19] C.F. Chyba \& C. Sagan, Endogenous production, exogenous delivery and impact-shock synthesis of organic molecules: an inventory for the origins of life, Nature 335 (1992) 125.

[20] K.A. Kvenvolden, J.G. Lawless, \& C. Ponnamperuna, Nonprotein amino acids in the Murchison meteorite, Proc. Natl. Acad. Sci. 68 (1971) 486.

[21] J.R. Cronin \& S. Pizzarello, Amino acid enantiomer excesses in meteorites: origin and significance, Adv. Space Res. 23 (1999) 293.

[22] G. Cooper, N. Kimmich, W. Belisle, et al., Carbonaceous meteorites as a source of sugar-related organic compounds for the early Earth, Nature 414 (2001) 879.

[23] L. d'Hendecourt, M. Jourdain de Muizon, E. Dartois, et al., ISO-SWS observations of solid state features towards RAFGL 7009S, Astron. Astrophys. 315 (1996) L365.

[24] D.C.B. Whittet, W.A. Schutte, A.G.G.M. Tielens, et al., An ISO SWS view of interstellar ices: First results, Astron. Astrophys. 315 (1996) L357.

[25] J.H. Lacy, H. Faraji, S.A. Sandford, \& L.J. Allamandola, Unraveling the 10 micron "silicate" feature of protostars: The detection of frozen interstellar ammonia, Astrophys. J. Lett. 501 (1998) L105.

[26] Y.-J. Chen, M. Nuevo, J.-M. Hsieh, et al., Carbamic acid produced by the UV/EUV irradiation of interstellar ice analogs, Astron. Astrophys. 464 (2007) 253.

[27] M. Nuevo, G. Auger, D. Blanot, \& L. d'Hendecourt, A detailed study of the amino acids produced from the vacuum UV irradiation of interstellar ice analogs, Orig. Life Evol. Biosph. 38 (2008) 37.

[28] M. Nuevo, S.N. Milam, S.A. Sandford, et al., Formation of uracil from the ultraviolet photo-irradiation of pyrimidine in pure $\mathrm{H}_{2} \mathrm{O}$ ices, Astrobiology 9 (2009) 683.

[29] M.P. Bernstein, J.P. Dworkin, S.A. Sandford, et al., Racemic amino acids from the ultraviolet photolysis of interstellar ice analogues, Nature 416 (2002) 401.

[30] G.M. Muñoz Caro, U.J. Meierhenrich, W.A. Schutte, et al., Amino acids from ultraviolet irradiation of interstellar ice analogues, Nature 416 (2002) 403.

[31] M. Nuevo, U.J. Meierhenrich, G.M. Muñoz Caro, et al., The effects of circularly polarized light on amino acid enantiomers produced by the UV irradiation of interstellar ice analogs, Astron. Astrophys. 457 (2006) 741.

[32] P. de Marcellus, C. Meinert, M. Nuevo, et al., Non-racemic amino acid production by ultraviolet irradiation of achiral interstellar ice analogs with circularly polarized light, Astrophys. J. Lett. 727 (2011a) L27.

[33] M. Nuevo, Y.-J. Chen, T.-S. Yih, et al., Amino acids formed from the UV/EUV irradiation of inorganic ices of astrophysical interest, Adv. Space Res. 40 (2007a) 1628.

[34] P.A. Gerakines, M.H. Moore, \& R. Hudson, Energetic processing of laboratory ice analogs: UV photolysis versus ion bombardment, J. Geophys. Res. 106 (2001) 33381. 
[35] E. Dartois, L. d'Hendecourt, W. Thi, et al., Combined VLT ISAAC/ISO SWS spectroscopy of two protostellar sources. The importance of minor solid state features, Astron. Astrophys. 394 (2002) 1057.

[36] E. Dartois, G.M. Muñoz Caro, D. Deboffle, \& L. d'Hendecourt, Diffuse interstellar medium organic polymers. Photoproduction of the 3.4, 6.85 and $7.25 \mu$ m features, Astron. Astrophys. 423 (2004) L33.

[37] G.M. Muñoz Caro \& W.A. Schutte, UV-photoprocessing of interstellar ice analogs: New infrared spectroscopic results, Astron. Astrophys. 412 (2003) 121.

[38] J.M. Greenberg, Chemical evolution in space - A source of prebiotic molecules, Adv. Space Res. 3 (1983) 19.

[39] J.P. Dworkin, D.W. Deamer, S.A. Sandford, \& L.J. Allamandola, Self-assembling amphiphilic molecules: Synthesis in simulated interstellar/precometary ices, Proc. Natl. Acad. Sci. 98 (2001) 815.

[40] J.P. Dworkin, J.S. Gillette, M.P. Bernstein, et al., An evolutionary connection between interstellar ices and IDPs? Clues from mass spectroscopy measurements of laboratory simulations, Adv. Space Res. 33 (2004) 67.

[41] E.L. Wagner \& D.F. Hornig, The vibrational spectra of molecules and complex ions in crystals III. Ammonium chloride and deutero-ammonium chloride J. Chem. Phys. 18 (1950) 296.

[42] M. Nuevo, S.N. Milam, S.A. Sandford, et al., XANES analysis of organic residues produced from the UV irradiation of astrophysical ice analogs, Adv. Space Res. 48 (2011) 1126.

[43] S.A. Sandford, J. Aléon, C.M.O'D. Alexander, et al., Organics captured from comet 81P/Wild 2 by the Stardust spacecraft, Science 314 (2006) 1720.

[44] G.D. Cody, H. Ade, C.M.O’D. Alexander, et al., Quantitative organic and light-element analysis of comet 81P/Wild 2 particles using C-, $N$-, and $O-\mu$-XANES, Meteorit. Planet. Sci. 43 (2008) 353.

[45] C.M.O'D. Alexander, M. Fogel, H. Yabuta, \& G.D. Cody, The origin and evolution of chondrites recorded in the elemental and isotopic compositions of their macromolecular organic matter, Geochim. Cosmochim. Acta 71 (2007) 4380.

[46] G.D. Cody, C.M.O'D. Alexander, H. Yabuta, et al., Organic thermometry for chondritic parent bodies, Earth Planet. Sci. Lett. 272 (2008) 446.

[47] J.P. Greenstein \& M. Winitz, Chemistry of the amino acids, John Wiley \& Sons Inc., New York, U.S.A. (1961) pp. 3-45.

[48] M. Nuevo, U.J. Meierhenrich, L. d'Hendecourt, et al., Enantiomeric separation of complex organic molecules produced from irradiation of interstellar/circumstellar ice analogs, Adv. Space Res. 39 (2007b) 400.

[49] Y.-J. Chen, M. Nuevo, T.-S. Yih, et al., Amino acids produced from the ultraviolet/extreme-ultraviolet irradiation of naphthalene in a $\mathrm{H}_{2} \mathrm{O}+\mathrm{NH}_{3}$ ice mixture, Month. Not. R. Astron. Soc. 384 (2008) 605.

[50] F. Combes, F., Nguyen-Q-Rieu, \& G. Wlodarczak, Search for interstellar glycine, Astron. Astrophys. 308 (1996) 618.

[51] Y.-J. Kuan, S.B. Charnley, H.-C. Huang, et al., Interstellar glycine, Astrophys. J. 593 (2003a) 848.

[52] L.E. Snyder, F.J. Lovas, J.M. Hollis, et al., A rigorous attempt to verify interstellar glycine, Astrophys. J. 619 (2005) 914.

[53] E.L. Shock \& M.D. Schulte, Summary and implications of reported amino acid concentrations in the Murchison meteorite, Geochim. Cosmochim. Acta 54 (1990) 3159. 
[54] M.A. Sephton, I.P. Wright, I. Gilmour, et al., High molecular weight organic matter in martian meteorites, Planet. Space Sci. 50 (2002) 711.

[55] Z. Martins, C.M.O'D. Alexander, G.E. Orzechowska, et al., Amino acid composition of primitive CR2 chondrites, Meteor. Planet. Sci. Suppl. 43 (2008a) 5195.

[56] M.H. Engel \& S.A. Macko, Isotopic evidence for extraterrestrial non-racemic amino acids in the Murchison meteorite, Nature 389 (1997) 265.

[57] J.R. Cronin \& S. Pizzarello, Enantiomeric excesses in meteoritic amino acids, Science 275 (1997) 951.

[58] S. Pizzarello, M. Zolensky, \& K.A. Turk, Nonracemic isovaline in the Murchison meteorite: Chiral distribution and mineral association, Geochim. Cosmochim. Acta 67 (2003) 1589.

[59] D.P. Glavin \& J.P. Dworkin, Enrichment in L-isovaline by aqueous alteration on CI and CM meteorite parent bodies, Proc. Natl. Acad. Sci. 106 (2009) 5487.

[60] J. Bailey, A. Chrysostomou, J.H. Hough, et al., Circular polarization in star-formation regions: Implications for biomolecular homochirality, Science 281 (1998) 672.

[61] T. Fukue, M. Tamura, R. Kandori, et al., Extended high circular polarization in the orion massive star forming region: Implications for the origin of homochirality in the Solar System, Orig. Life Evol. Biosph. arXiv reference: 1001.2608 (2010).

[62] M. Nuevo, J.H. Bredehöft, U.J. Meierhenrich, et al., Urea, glycolic acid, and glycerol in an organic residue produced by ultraviolet irradiation of interstellar/pre-cometary ice analogs, Astrobiology $\mathbf{1 0}$ (2010) 245.

[63] J.R. Cronin \& S. Chang, Chemistry of life's origins, J.M. Greenberg, V. Pirronello, \& C. Mendoza-Gómez, Eds., Kluwer, Dordrecht, the Netherlands (1993) pp. 209-258.

[64] G.W. Cooper \& J.R. Cronin, Linear and cyclic aliphatic carboxamides of the Murchison meteorite: Hydrolyzable derivatives of amino acids and other carboxylic acids, Geochim. Cosmochim. Acta 59 (1995) 1003.

[65] P. de Marcellus, M. Bertrand, M. Nuevo, et al., Prebiotic significance of extraterrestrial ice photochemistry: Detection of hydantoin in organic residues, Astrobiology 11 (2011b) 847.

[66] A. Shimoyama \& R. Ogasawara, Dipeptides and diketopiperazines in the Yamato-791198 and Murchison carbonaceous chondrites, Orig. Life Evol. Biosph. 32 (2002) 165.

[67] A. Commeyras, J. Taillades, H. Collet, et al., Dynamic co-evolution of peptides and chemical energetics, a gateway to the emergence of homochirality and the catalytic activity of peptides, Orig. Life Evol. Biosph. 34 (2004) 35.

[68] G. Danger, L. Boiteau, H. Cottet, \& R. Pascal, The peptide formation mediated by cyanate revisited. $N$-carboxyanhydrides as accessible intermediates in the decomposition of $N$-carbamoylamino acids, J. Am. Chem. Soc. 128 (2006) 7412.

[69] D. Deamer, J.P. Dworkin, S.A. Sandford, et al., The first cell membranes, Astrobiology 2 (2002) 371.

[70] M.N. Simon \& M. Simon, Search for interstellar acrylonitrile, pyrimidine, and pyridine, Astrophys. J. 184 (1973) 757.

[71] Y.-J. Kuan, C.-H. Yan, S.B. Charnley, et al., A search for interstellar pyrimidine, Monthly Not. R. Astron. Soc. 345 (2003b) 650. 
[72] S.B. Charnley, Y.-J. Kuan, H.-C. Huang, et al., Astronomical searches for nitrogen heterocycles, Adv. Space Res. 36 (2005) 137.

[73] E. Peeters, A.L. Mattioda, D.M. Hudgins, \& L.J. Allamandola, Polycyclic aromatic hydrocarbon emission in the 15-21 micron region, Astrophys. J. 617 (2004) L65.

[74] C.E. Folsome, J. Lawless, M. Romiez, \& C. Ponnamperuma, Heterocyclic compounds indigenous to the Murchison meteorite, Nature 232 (1971) 108.

[75] R. Hayatsu, E. Anders, M.H. Studier, \& L.P. Moore, Purines and triazines in the Murchison meteorite, Geochim. Cosmochim. Acta 39 (1975) 471.

[76] W. van der Velden \& A. Schwartz, Search for purines and pyrimidines in the Murchison meteorite, Geochim. Cosmochim. Acta 41 (1977) 961.

[77] M.P. Callahan, K.E. Smith, H.J. Cleaves II, et al., Carbonaceous meteorites contain a wide range of extraterrestrial nucleobases, Proc. Natl. Acad. Sci. 108 (2011) 13995.

[78] P. Stoks \& A. Schwartz, Uracil in carbonaceous meteorites, Nature 282 (1979) 709.

[79] Z. Martins, O. Botta, M.L. Fogel, et al., Extraterrestrial nucleobases in the Murchison meteorite, Earth Plan. Sci. Lett. 270 (2008b) 130.

[80] A. Ricca, C.W. Bauschlicher, \& E.L.O. Bakes, A computational study of the mechanisms for the incorporation of a nitrogen atom into polycyclic aromatic hydrocarbons in the Titan haze, Icarus $\mathbf{1 5 4}$ (2001) 516.

[81] M. Nuevo, S.N. Milam, \& S.A. Sandford, Nucleobases and prebiotic molecules in organic residues produced from the ultraviolet photo-irradiation of pyrimidine in $\mathrm{NH}_{3}$ and $\mathrm{H}_{2} \mathrm{O}+\mathrm{NH}_{3}$ ices, Astrobiology 12 (2012) in press.

[82] P.P. Bera, M. Nuevo, S.N. Milam, et al., Mechanism for the abiotic synthesis of uracil via UV-induced oxidation of pyrimidine in pure $\mathrm{H}_{2} \mathrm{O}$ ices under astrophysical conditions, J. Chem. Phys. 133 (2010) 104303.

[83] J.H. Lacy, F. Baas, L.J. Allamandola, et al., 4.6 micron absorption features due to solid phase CO and cyano group molecules toward compact infrared sources, Astrophys. J. 276 (1984) 533.

[84] K. Demyk, E. Dartois, L. d'Hendecourt, et al., Laboratory identification of the $4.62 \mu \mathrm{m}$ solid state absorption band in the ISO-SWS spectrum of RAFGL 7009S, Astron. Astrophys. 339 (1998) 553.

[85] L.P. Keller, S. Bajt, G.A. Baratta, et al. Infrared spectroscopy of Comet 81P/Wild 2 samples returned by Stardust, Science 314 (2006) 1728.

[86] G.J. Flynn, L.P. Keller, M. Feser, et al., The origin of organic matter in the solar system: evidence from the interplanetary dust particles, Geochim. Cosmochim. Acta 67 (2003) 4791.

[87] S. Wirick, G.J. Flynn, L.P. Keller, et al. Organic matter from comet 81P/Wild 2, IDPs, and carbonaceous meteorites; similarities and differences, Meteor. Planet. Sci. 44 (2009) 1611.

[88] M. Abe, Y. Takagi, K. Kitazato, et al., Near-Infrared Spectral Results of Asteroid Itokawa from the Hayabusa Spacecraft, Science 312 (2006) 1334.

[89] P. Jenniskens, M.H. Shaddad, D. Numan, et al., The impact and recovery of asteroid $2008 \mathrm{TC}_{3}$, Nature 458 (2009) 485.

[90] D.P. Glavin, A.D. Aubrey, M.P. Callahan, et al., Extraterrestrial amino acids in the Almahata Sitta meteorite, Meteor. Planet. Sci. 45 (2010) 1695. 
[91] A.S. Burton, D.P. Glavin, M.P. Callahan, et al., Heterogeneous distributions of amino acids provide evidence of multiple sources within the Almahata Sitta parent body, asteroid $2008 \mathrm{TC}_{3}$, Meteor. Planet. Sci. 46 (2011) 1703.

[92] W.H.-P. Thiemann \& U. Meierhenrich, ESA mission ROSETTA will probe for chirality of cometary amino acids, Orig. Life Evol. Biosph. 31 (2001) 199.

[93] F. Goesmann, H. Rosenbauer, R. Roll, et al., COSAC, the cometary sampling and composition experiment on Philae, Space Sci. Rev. 128 (2007) 257. 